\title{
Баллистическое течение двумерных электронов в магнитном поле
}

\author{
() А.Н. Афранасьев, П.С. Алексеев, А.А. Грешнов, М.А. Семина \\ Физико-технический институт им. А.Ф. Иофрфе Российской академии наук, \\ 194021 Санкт-Петербург, Россия \\ E-mail: Afanasiev.An@mail.ioffe.ru
}

Поступила в Редакцию 26 февраля 2021 г. В окончательной редакции 1 марта 2021 г. Принята к публикации 1 марта 2021 г.

В проводниках с очень малой плотностью дефектов электроны при низких температурах сталкиваются преимущественного с краями образца, поэтому перенос заряда и тепла осуществляется баллистически. Приложение перпендикулярного магнитного поля существенно меняет характер баллистического транспорта. Для случая двумерных (2D) электронов при магнитных полях таких, что диаметр циклотронных траекторий становится меньше ширины образца, происходит формирование гидродинамического режима транспорта. В таком режиме течение в основном контролируется редкими межэлектронными столкновениями, определяющими эффект вязкости. В настоящей работе мы изучаем баллистическое течение $2 \mathrm{D}$ электронов в длинных образцах в магнитных полях вплоть до критического поля перехода в гидродинамический режим. Из решения кинетического уравнения мы получаем аналитические выражения для профилей плотности тока и холловского электрического поля при магнитных полях вдали и вблизи от баллистическо-гидродинамического перехода, а также для продольного и холловского сопротивлений в этих диапазонах. Наши теоретические результаты, по-видимому, описывают наблюдавшееся продольное сопротивление чистых графеновых образцов в диапазоне магнитных полей ниже баллистическо-гидродинамического перехода.

Ключевые слова: двумерные электроны, высокая подвижность, баллистический режим транспорта, магнетосопротивление, эффект Холла.

DOI: $10.21883 /$ FTP.2021.07.51019.9640

\section{1. Введение}

В достаточно малых образцах чистых двумерных и трехмерных проводников электроны при очень низких температурах чаще всего сталкиваются с краями образца. В таком случае электронный транспорт является баллистическим. С повышением температуры электронэлектронные столкновения могут приводить к формированию вязкой электронной жидкости и реализации гидродинамического режима транспорта. Несмотря на то что теория вязкой электронной жидкости активно развивается в течение длительного времени [1-3], несомненные экспериментальные свидетельства формирования такой жидкости были получены лишь недавно в высококачественном графене, вейлевских полуметаллах и квантовых ямах на основе GaAs [4-25]. Вывод о наличии гидродинамического течения в этих экспериментах был сделан по наблюдениям: специфической зависимости среднего сопротивления образца от ширины образца [4], нелокального отрицательного сопротивления [5-7], гигантского отрицательного магнетосопротивления [12-20] и магнитного резонанса при двойной циклотронной частоте [21-25]. Экспериментальная реализация гидродинамического транспорта привела к развитию его теории в новых направлениях (см., например, работы [26-33]).

В недавних работах $[10,11]$ было измерено пространственное распределение плотности тока и холловского электрического поля в потоке двумерных электронов в полосках графена. В работе [10] наблюдение эволюции кривизны профиля холловского поля послужило свидетельством перехода между баллистическим и гидродинамическим режимами транспорта. В [11] плотность тока гидродинамического и омического течений в узкой полоске была определена при помощи измерения распределения локального магнитного поля, наведенного током в полоске.

В работах $[34,35]$ течение взаимодействующих двумерных (2D) электронов в узком баллистическом образце было теоретически исследовано в пределе слабых магнитных полей путем аналитического решения упрощенного кинетического уравнения. Было показано, что при низких температурах холловское электрическое поле почти во всем образце (за исключением малых окрестностей краев) равно половине от его „обычной величины“ в макроскопических омических образцах. Было также показано, что межчастичные столкновения, во-первых, контролируют максимальную длину баллистических траекторий, которая определяет величины баллистического тока и холловского поля, и, во-вторых, приводят к малым гидродинамическим поправкам, которые являются предвестниками формирования вязкого течения.

В работе [36] переход между баллистическим и гидродинамическим режимами транспорта для пуазейлевского течения 2D электронов в перпендикулярном магнитном поле был теоретически исследован при помощи численного решения кинетического уравнения. При величине магнитного поля $B=B_{c}$, при которой диаметр циклотронной орбиты электрона $2 R_{c}$ становится равным 
ширине образца $W$ и некоторые электроны начинают делать полный оборот без рассеяния на краях, продольное и холловское сопротивления как функции магнитного поля испытывают скачок. В работе [37] пуазейлевское течение двумерных электронов в присутствии магнитного поля было исследовано более детально, а именно были численно рассчитаны распределения холловского поля по сечению образца при $B<B_{c}$ и $B>B_{c}$, а также построено аналитическое решение кинетического уравнения в баллистической области $B<B_{c}$. Это позволило объяснить эволюцию кривизны профиля холловского поля с изменением магнитного поля, наблюдавшуюся в эксперименте [10]. В работе [38] была развита аналитическая теория перехода между баллистическим и гидродинамическим режимами транспорта в магнитном поле при $\left|B-B_{c}\right| \ll B_{c}$. Показано, что для течения двумерных электронов в образцах высокого качества этот переход является резким фазовым переходом. Было дано описание характера динамики электронов в окрестности перехода $\left|B-B_{c}\right| \ll B_{c}$, и была построена модель среднего поля для количественного описания перехода.

Цель настоящей работы - теоретическое изучение баллистического транспорта взаимодействующих 2D электронов в длинных образцах в магнитных полях $B$, меньших поля $B_{c}$ перехода в гидродинамический режим. Используя общее аналитическое решение кинетического уравнения в баллистическом режиме, полученное в работах [37,38], мы показываем, что в диапазоне полей $B_{1}<B<B_{2}$ (точнее, $B \gg B_{1}$ и $B_{c}-B \gg B_{c}-B_{2}$ ) течение электронов при низкой температуре в основном определяется рассеянием электронов на краях образца и влиянием циклотронного эффекта магнитного поля. Слабые межэлектронные столкновения с интенсивностью $\gamma$ определяют величины $B_{1}$ и $B_{2}$ $\left(B_{1} \propto \gamma^{2}\right.$ и $\left.B_{c}-B_{2} \propto \gamma\right)$ и приводят только к малым поправкам ко всем характеристикам течения в этом диапазоне.

Мы рассчитываем плотность тока $j(y)$ и холловское поле $E_{\mathrm{H}}(y)$, а также продольное и холловское сопротивления, $\rho_{x x}(B)$ и $\rho_{x y}(B)$. Профиль плотности тока $j(y)$ с ростом магнитного поля от $B_{1}$ до $B_{2}$ эволюционирует от почти плоского до деформированной полуокружности. Полученные профили холловского поля $E_{\mathrm{H}}(y)$, как при малых полях, $B_{1} \ll B \ll B_{c}$, так и вблизи перехода, $B_{c}-B_{2} \ll B_{c}-B \ll B_{c}$, сильно отличаются от плоского и расходятся около краев образца: $E_{\mathrm{H}}(y) \neq$ const и $E_{\mathrm{H}}(y) \rightarrow \infty$ при $y \rightarrow \pm W / 2$. Амплитуда холловского поля становится независимой от магнитного поля $B$ в области умеренно малых полей, $B_{1} \ll B \ll B_{c}$, поэтому величина $\rho_{x y}(B)$ имеет в этой области вид, похожий на плато. Такое поведение $\rho_{x y}(B)$, по-видимому, является одной из форм баллистической аномалии магнетосопротивления, которая ранее наблюдалась экспериментально и была получена в численном моделировании в двумерных образцах с 4 контактами [39]. Зависимости от магнитного поля сопротивле- ний, $\rho_{x x}(B), \rho_{x y}(B)$, и их производных по $B$ во всем диапазоне $B_{1}<B<B_{2}$ проявляют нетривиальное немонотонное поведение и согласуются с результатами численного моделирования [36]. Теоретический результат для $\rho_{x x}(B)$, по-видимому, соответствует зависимостям сопротивления высококачественных образцов графена и квантовых ям GaAs, наблюдавшимся в эксперимен$\operatorname{Tax}[10,11,18]$.

\section{2. Модель}

Рассмотрим течение двумерных вырожденных электронов в длинном образце (длиной $L$ ) с шероховатыми краями при низкой температуре. Рассеяние электронов на краях предполагается диффузионным: импульс отраженных электронов распределен изотропно независимо от импульса налетающих электронов. В объеме образца электроны редко рассеиваются друг на друге и (или) на беспорядке (см. рис. 1,a).

Наш подход позволяет рассматривать системы, являющиеся смешением двух предельных случаев: внутри образца нет дефектов и электроны в объеме рассеиваются только друг на друге с сохранением импульса;
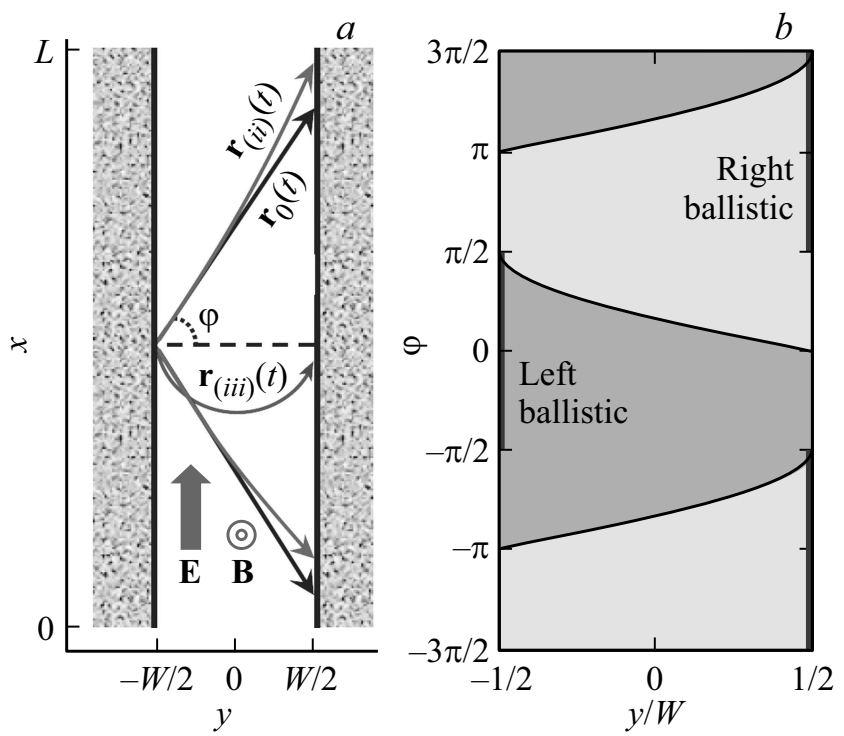

Рис. 1. $a-$ длинный баллистический образец в магнитном поле, прямые $\mathbf{r}_{0}(t)$ - траектории электронов в нулевом магнитном поле; $\mathbf{r}_{(i i)}(t)$ - траектории в слабых полях, отвечающих аномалии в магнетосопротивлении (вторая баллистическая подобласть); $\mathbf{r}_{(i i i)}(t)$ - траектории в относительно сильных магнитных полях, с нижней стороны приближающихся к фазовому переходу между баллистическим и гидродинамическим режимами (третья баллистическая подобласть). $b-$ области в плоскости координат $(y, \varphi)$, где двигаются преимущественно электроны, отраженные от левого и правого краев образца; вертикальные толстые линии показывают положение электронов, только что отраженных от левого и правого краев; линии на границах областей соответствуют траекториям, касающимся краев образца под углами $\varphi= \pm \pi / 2$. 
межчастичные столкновения отсутствуют, при этом происходит рассеяние электронов на беспорядке в объеме образца, что приводит к слабой релаксации импульса. Мы предполагаем темп любого рассеяния в объеме образца медленным: $W \ll l$, где $l=v_{\mathrm{F}} / \gamma-$ средняя длина свободного пробега электрона по отношению ко всем механизмам рассеяния в объеме образца, $\gamma$ суммарный темп рассеяния, $v_{\mathrm{F}}-$ скорость электронов на уровне Ферми.

В слабых магнитных полях, $B<B_{c}$, когда диаметр циклотронной орбиты больше ширины образца, $2 R_{c}>W$, каждый электрон преимущественно рассеивается на краях образца. Следовательно, в главном порядке по $\gamma$ в таких магнитных полях режим рассеяния электронов, вообще говоря, является баллистическим. При этом рассеяние в объеме может приводить к ограничению времени, проводимого электронами на баллистических траекториях [34,35], и к небольшим поправкам к параметрам чисто баллистического течения (совсем не учитывающего межэлектронные столкновения) [38].

В сильных магнитных полях, $B>B_{c}$, соответствующих $W>2 R_{c}$, электроны по характеру их динамики разделяются на две группы: „краевые“ электроны, которые двигаются по „скачущим“ траекториям, в основном рассеиваясь только на одном из краев образца, и „центральные“ электроны, траектории которых не касаются краев [36,38]. Вблизи от поля перехода, $B-B_{c} \ll B_{c}$, центры траекторий „центральных“ электронов лежат в малом диапазоне координат $y$ вблизи центра образца, $|y| \ll W$, поэтому их относительная доля много меньше доли „краевых“ электронов, и, следовательно, они рассеиваются в основном на „краевых“ электронах (и/или на объемном беспорядке). Появление фазы „центральных“ электронов есть начальная стадия формирования фазы объемных (двумерных) электронов, отвечающей за гидродинамический/омический транспорт при $W \gg R_{c}[38]$.

Мы будем изучать линейный отклик двумерных электронов на однородное электрические поле $\mathbf{E}_{0} \| x$ в присутствии внешнего магнитного поля $\mathbf{B}$, перпендикулярного плоскости образца (см. рис. $1, a)$. Соответствующая функция распределения двумерных электронов приобретает неравновесную часть: $\delta f(y, \mathbf{p})=-f_{\mathrm{F}}^{\prime}(\varepsilon) f(y, \varphi, \varepsilon) \propto E_{0}$, где $f_{\mathrm{F}}(\varepsilon)-$ функция распределения Ферми, $\varepsilon$ - энергия электрона, $\varphi-$ угол между скоростью электрона $\mathbf{v}=v(\varepsilon)[\sin \varphi, \cos \varphi]$ и нормалью к левому краю образца, $\mathbf{p}=m \mathbf{v}-$ импульс электрона, $m$ - масса электрона (см. рис. 1, $a$ ). Зависимость $\delta f$ от координаты вдоль образца $x$ отсутствует, так как $L \gg W$. Мы также пренебрегаем зависимостью скорости электрона $v(\varepsilon)=|\mathbf{v}(\varepsilon)|$ и неравновесной части функции распределения $\delta f(y, \mathbf{p})$ от энергии электрона. Подобное упрощение допустимо для вырожденного распределения электронов. Далее мы используем единицы измерения, в которых характерная скорость электрона $v(\varepsilon) \equiv v_{\mathrm{F}}$ и заряд электрона $e$ положены равными 1 . В выбранных единицах координата, время, и обратное электрическое поле, $1 / E_{0}$, имеют одинаковую размерность.

Кинетическое уравнение для неравновесной функции распределения $f(y, \varphi, \varepsilon)$ принимает вид

$$
\cos \varphi \frac{\partial f}{\partial y}-\sin \varphi E_{0}-\cos \varphi E_{\mathrm{H}}-\omega_{c} \frac{\partial f}{\partial \varphi}=\operatorname{St}[f],
$$

где $\omega_{c}=e B / m c-$ циклотронная частота, $E_{\mathrm{H}}=E_{\mathrm{H}}(y)-$ холловское электрическое поле, возникающее за счет перераспределения электронов в магнитном поле, интеграл столкновений $\mathrm{St}[f]$ описывает как сохраняющие импульс электрон-электронные столкновения, так и рассеяние на объемном беспорядке, приводящие к релаксации импульса.

В данной работе используются упрощенный вид интегралов электрон-электронных столкновений и рассеяния на беспорядке (что позволяет получить асимптотически точное (по $\gamma W \ll 1)$ аналитическое решение кинетического уравнения при $\left.B<B_{c}\right)$ :

$$
\mathrm{St}[f]=-\gamma f+\gamma_{e e} \hat{P}_{e e}[f]+\gamma_{d} \hat{P}_{d}[f],
$$

где $\gamma_{e e}$ и $\gamma_{d}$ - темпы электрон-электронного рассеяния и рассеяния на беспорядке, $\gamma=\gamma_{e e}+\gamma_{d}-$ суммарный темп рассеяния, $\hat{P}$ и $\hat{P}_{0}-$ проекционные операторы для функций $f(\varphi)$ на подпространствах $\left\{1, e^{ \pm i \varphi}\right\}$ и $\{1\}$ соответственно. Такой интеграл столкновений сохраняет возмущения функции распределения, отвечающие неравновесной плотности. Интеграл (2) при $\gamma_{d}=0$ описывает также сохранение импульса в процессе межчастичных столкновений. Такой вид St использовался, в частности, в работах [34,35] для исследования баллистического транспорта двумерных взаимодействующих электронов в пределе $B \rightarrow 0$ и в работе [38] для построения теории среднего поля, описывающей фазовый переход между баллистическим и гидродинамическим режимами транспорта вблизи $B=B_{c}$.

Мы предполагаем продольные края образца полностью шероховатыми, поэтому рассеяние электронов на краях является диффузионным и граничные условия для функции распределения имеют вид [35,38,39]: $f(-W / 2, \varphi)=c_{l}$ при углах $-\pi / 2<\varphi<\pi / 2$ и $f(W / 2, \varphi)=c_{r}$ при углах $\pi / 2<\varphi<3 \pi / 2$ (см. также рис. $1, a, b)$, где величины $c_{l}=c_{l}[f]$ и $c_{r}=c_{r}[f]$ пропорциональны $y$-компонентам налетающего потока электронов на левом $(y=-W / 2)$ и правом $(y=W / 2)$ краях образца:

$$
\begin{gathered}
c_{l}=-\frac{1}{2} \int_{\pi / 2}^{3 \pi / 2} d \varphi^{\prime} \cos \varphi^{\prime} f\left(-W / 2, \varphi^{\prime}\right), \\
c_{r}=\frac{1}{2} \int_{-\pi / 2}^{\pi / 2} d \varphi^{\prime} \cos \varphi^{\prime} f\left(W / 2, \varphi^{\prime}\right) .
\end{gathered}
$$

Граничные условия (3) означают, что вероятность отражения электрона от шероховатого края не зависит 
от угла рассеяния $\varphi$ и поперечная компонента потока электронов, $j_{y}(y)=\left[n_{0} /(\pi m)\right] \int_{0}^{2 \pi} d \varphi^{\prime} \cos \varphi^{\prime} f\left(y, \varphi^{\prime}\right)$, равна нулю на краях, $j_{y}(y= \pm W / 2)=0$ (и тем самым везде в образце вследствие уравнения неразрывности $\left.\operatorname{div} \mathbf{j}=j^{\prime}=0\right)$.

Плотность тока вдоль образца, $j(y) \equiv j_{x}(y)$, вычисляется по формуле

$$
j(y)=\frac{n_{0}}{\pi m} \int_{0}^{2 \pi} d \varphi^{\prime} \sin \varphi^{\prime} f\left(y, \varphi^{\prime}\right) .
$$

Если электрический ток течет через образец во внешнем магнитном поле, то под действием силы Лоренца возникают возмущение плотности заряда и холловское электрическое поле. Оба эффекта описываются нулевой $(m=0)$ угловой гармоникой функции распределения:

$$
f^{m=0}(y)=\frac{1}{2 \pi} \int_{0}^{2 \pi} d \varphi^{\prime} f\left(y, \varphi^{\prime}\right) .
$$

На рис. $1, b$ показаны области на плоскости $(y, \varphi)$, отвечающие баллистическому движению электронов, отраженных от правого и от левого краев образца. В узких образцах, $W \ll R_{c}$, „левая“ и „правая“ баллистические области по форме близки к прямоугольникам: $\quad[-\pi / 2, \pi / 2] \times[-W / 2, W / 2] \quad$ и $[\pi / 2,3 \pi / 2] \times[-W / 2, W / 2]$. В более широких образцах, $W \sim R_{c}, \quad W<2 R_{c}, \quad$ границы ,левой“ и „правой“ областей (кривые $\varphi_{+}(y)$ и $\pm \pi+\varphi_{-}(y)$, где $\left.\varphi_{ \pm}(y)=\arcsin \left[1-\omega_{c}(W / 2 \pm y)\right]\right)$ начинают существенно зависеть от координаты $y$ (см. рис. $1, b$ ). Линии границ совпадают с траекториями электронов, падающих на края тангенциально по касательной к краю. Такие электроны на пограничных траекториях не описываются приведенными выше граничными условиями. Следовательно, функция распределения $f(y, \varphi)$ не определена в точках $\varphi_{+}(y), \pm \pi+\varphi_{-}(y)$ и поэтому может иметь разрыв или другую сингулярность в этих точках.

Кинетическое уравнение (1) удобно переписать в виде

$$
\begin{aligned}
{\left[\cos \varphi \frac{\partial}{\partial y}+\gamma\right] \tilde{f} } & -\sin \varphi E_{0} \\
& =\gamma_{e e} \hat{P}_{e e}[\tilde{f}]+\gamma_{d} \hat{P}_{d}[\tilde{f}]+\omega_{c} \frac{\partial \tilde{f}}{\partial \varphi}
\end{aligned}
$$

где введена функция $\tilde{f}(y, \varphi)=f(y, \varphi)+\phi(y)$, уходный член интеграла столкновений был перенесен из правой части в левую. Здесь $\phi-$ электростатический потенциал холловского электрического поля: $E_{\mathrm{H}}=-\phi^{\prime}$. Действительно, как следует из уравнения (1), холловский потенциал $\phi(y)$ играет ту же роль в транспортном уравнении, что и нулевая гармоника функции распределения (которая пропорциональна плотности электронов и с которой он связан уравнениями электростатики). Таким образом, для учета $\phi(y)$ и $f^{m=0}(y)$ в рамках одного подхода удобно ввести функцию $\tilde{f}(y, \varphi)=f(y, \varphi)+\phi(y)$.
Нулевая гармоника $\tilde{f}^{m=0}(y)$ обобщенной функции распределения $\tilde{f}$ имеет вид $\tilde{f}^{m=0}(y)=\delta \mu(y)+\phi(y)$, где $\delta \mu(y)$ - возмущение химического потенциала электронов. Для стационарного (и достаточно медленного) течения величины $\delta \mu(y)$ и $\phi(y)$ связаны электростатическими соотношениями [25]. Для рассмотренного случая двумерного однокомпонентного электронного газа электростатический потенциал $\phi$ обычно существенно больше, чем соответствующее возмущение химического потенциала $\delta \mu$ [25]. Поэтому холловское электрическое поле может быть вычислено по простой формуле $E_{\mathrm{H}}(y) \approx-\tilde{f}^{m=0^{\prime}}(y)$.

Для краткости мы в дальнейшем опускаем знак „тильда“ функции $\tilde{f}$ и пишем просто $f \equiv \tilde{f}$.

\section{3. Общее решение в баллистическом режиме}

Изучим баллистический транспорт, который, как отмечалось выше, реализуется в относительно слабых магнитных полях, $B<B_{c}$, в которых диаметр циклотронной орбиты электрона больше ширины образца, $2 R_{c}>W$.

B работах $[37,38]$ было показано, что при наличии слабого межэлектронного рассеяния почти во всех магнитных полях меньше критического, $B<B_{c}$, а именно при $2-\omega_{c} W \gg \gamma W$, течение электронов действительно является баллистическим. При этом в главном порядке по темпу рассеяния $\gamma$ такие течения описываются кинетическим уравнением (6) с опущенным приходным членом [37,38]:

$$
\left[\cos \varphi \frac{\partial}{\partial y}+\gamma\right] f-\sin \varphi E_{0}=\omega_{c} \frac{\partial f}{\partial \varphi} .
$$

Анализ уравнения (7) показал, что баллистический режим разделяется на три подрежима [38]. Переход между ними сопровождается эволюцией профилей плотности тока $j(y)$ и холловского электрического поля $E_{\mathrm{H}}(y)$, a также изменением типа зависимостей продольного, $\rho_{x x}(B)$, и холловского, $\rho_{x y}(B)$, сопротивлений от магнитного поля.

Эти три подрежима имеют следующий вид.

1) $\omega_{c} \ll \gamma^{2} W$. Длина максимального сегмента циклотронной орбиты, который может быть вписан в полоску $l_{b}^{(2)}=\sqrt{R_{c} W}$, больше средней длины свободного пробега электронов относительно объемного рассеяния: $l_{b}^{(2)} \gg l=1 / \gamma$. Поэтому объемное рассеяние определяет для большинства электронов эффективную длину баллистической траектории: $l_{b}^{(1)} \sim l$.

2) $\gamma^{2} W \ll \omega_{c} \ll 1 / W$. В этом подрежиме, напротив, $l_{b}^{(2)} \ll l$, при этом магнитный параметр $\omega_{c} W$ мал. Максимальная длина баллистической траектории определяется геометрией расположения траекторий и поэтому равна $l_{b}^{(2)}$.

3) $\omega_{c} \sim 1 / W$ при условии, что $2-\omega_{c} W \gg \gamma W$. В этом случае так же $l_{b}^{(2)} \ll l$, но магнитный параметр $\omega_{c} W$ 
имеет величину порядка $1, \omega_{c} W \sim 1$, или близок к критическому значению, $\omega_{c} W=2$. Средняя длина баллистической траектории $l_{b}^{(3)}$ также определяется геометрией траекторий электрона и равна по порядку величины $W$.

Аналитическое решение кинетического уравнения (7), описывающее все три подрежима 1)-3), было получено в работах $[37,38]$ при помощи метода характеристик для обыкновенных дифференциальных уравнений. Полученное решение $f(y, \varphi)$ представляет собой разрывную функцию с областями непрерывности, показанными на рис. $1, b$. Для электронов, отраженных от левого и правого краев образца, траектории которых лежат в диапазонах $-\pi+\varphi_{-}(y)<\varphi<\varphi_{+}(y)$ и $\varphi_{+}(y)<\varphi<\pi+\varphi_{-}(y)$ соответственно, мы используем следующие обозначения: $f(y, \varphi)=f_{ \pm}(y, \varphi)$ (см. рис. $\left.1, b\right)$. Две компоненты $f_{ \pm}(y, \varphi)$ функции $f$ имеют вид

$$
\begin{aligned}
f_{ \pm} & (y, \varphi)=\frac{E_{0}}{\gamma^{2}+\omega_{c}^{2}} \\
& \times\left[\omega_{c} \cos \varphi+\gamma \sin \varphi+e^{\gamma \varphi / \omega_{c}} Z_{ \pm}\left(\sin \varphi+\omega_{c} y\right)\right],
\end{aligned}
$$

где не зависящие от координаты $y$ вклады $\omega_{c} \cos \varphi$ и $\gamma \sin \varphi$ являются частными решениями уравнения (7), отвечающими формулам Друде (в случае, когда темп рассеяния $\gamma$ отвечает рассеянию только на беспорядке). Вклад $e^{\gamma \varphi / \omega_{c}} Z_{ \pm}(X)$ есть общее решение кинетического уравнения (7) без полевого члена $\sin \varphi E_{0}$, удовлетворяющее правильным граничным условиям с ненулевыми значениями параметров $c_{l, r}(3)$.

Подставляя выражение (8) в граничные условия $f(y=\mp W / 2)=c_{l, r}$, мы получаем явный вид $Z_{ \pm}(X)[38]$ :

$$
\begin{aligned}
& Z_{+}(X)=e^{-\gamma \frac{\arcsin \left(X+\omega_{c} W / 2\right)}{\omega_{c}}} \\
& \times\left[c_{l}-\gamma\left(X+\omega_{c} W / 2\right)-\omega_{c} \sqrt{1-\left(X+\omega_{c} W / 2\right)^{2}}\right]
\end{aligned}
$$

И

$$
\begin{aligned}
& Z_{-}(X)=e^{-\gamma \frac{\pi-\arcsin \left(X-\omega_{c} W / 2\right)}{\omega_{c}}} \\
& \times\left[c_{r}-\gamma\left(X-\omega_{c} W / 2\right)+\omega_{c} \sqrt{1-\left(X-\omega_{c} W / 2\right)^{2}}\right],
\end{aligned}
$$

где коэффициенты $c_{l}$ и $c_{r}$ определены из балансных граничных соотношений $j_{y}(y=\mp W / 2)=0$, принимающих вид

$$
\left(\begin{array}{ll}
I_{l l} & I_{l r} \\
I_{r l} & I_{r r}
\end{array}\right)\left(\begin{array}{l}
c_{l} \\
c_{r}
\end{array}\right)=-\left(\begin{array}{c}
I_{l} \\
I_{r}
\end{array}\right) .
$$

В этом уравнении коэффициенты первой строки матрицы выражаются в виде интегралов

$$
I_{l l}=2+\int_{-\pi+\varphi_{0}}^{-\pi / 2} d \varphi \cos \varphi e^{\gamma \frac{\pi+2 \varphi}{\omega_{c}}}
$$

и

$$
I_{l r}=\int_{\pi / 2}^{\pi+\varphi_{0}} d \varphi \cos \varphi e^{\gamma \frac{\varphi-\pi+\arcsin \left(\sin \varphi-\omega_{c} W\right)}{\omega_{c}}}
$$

а первая компонента вектора в правой части есть

$$
\begin{aligned}
I_{l} & =\frac{\pi \omega_{c}}{2}+\int_{\varphi_{0}}^{\pi / 2} d \varphi \cos \varphi e^{\gamma \frac{2 \varphi-\pi}{\omega_{c}}}\left(\omega_{c} \cos \varphi-\gamma \sin \varphi\right) \\
& -\int_{-\pi / 2}^{\varphi_{0}} d \varphi \cos \varphi e^{\gamma \frac{\varphi-\arcsin \left(\sin \varphi+\omega_{c} W\right)}{\omega_{c}}} \\
& \times\left[\omega_{c} \sqrt{1-\left(\sin \varphi+\omega_{c} W\right)^{2}}+\gamma\left(\sin \varphi+\omega_{c} W\right)\right] .
\end{aligned}
$$

Остальные коэффициенты уравнения (11), $I_{r r}, I_{r l}$ и $I_{r}$, связаны с $I_{l l}, I_{l r}$ и $I_{l}$ посредством формул $I_{r r}=-I_{l l}$, $I_{r l}=-I_{l r}, I_{r}=I_{l}$. В выражениях (12)-(14) введено обозначение $\varphi_{0}=\arcsin \left(1-\omega_{c} W\right)$.

При произвольном значении параметра $\omega_{c} W$ интегралы (12)-(14) могут быть определены лишь численно. Тем не менее явные выражения для них и, как следствие, для величин $c_{l, r}$ и $j(y), E_{\mathrm{H}}(y)$ могут быть получены аналитически в следующих предельных случаях: в подобласти 1) баллистического режима, $\omega_{c} \ll \gamma^{2} W$ (см. работы $[35,38]) ;$ в подобласти 2) баллистического режима, $\gamma^{2} W \ll \omega_{c} \ll 1 / W$ (см. работы $[37,38]$ и разд. 4 данной статьи); в правой сингулярной части подобласти 3) баллистического режима, $\gamma W \ll 2-\omega_{c} W \ll 1$ (см. работу [38] и разд. 4 данной статьи).

Подрежим 1) был подробно исследован в работах $[34,35,38]$ при помощи решения кинетического уравнения (7) по методу последовательных приближений, а также при помощи анализа точного решения (8). В этом подрежиме внешнее магнитное поле вносит лишь малые поправки в центральную часть течения в области $|W / 2-| y \| \gg \omega_{c} / \gamma^{2}$, но в то же время приводит к не описываемому теорией возмущений течению электронов в прикраевых областях образца $|W / 2-| y \| \lesssim \omega_{c} / \gamma^{2}$.

Подрежимы 2) и 3) были частично исследованы в работах $[37,38]$. Ниже при помощи общего решения (8) мы получим для течений в диапазонах 2) и 3) новые результаты, а именно аналитически вычисляем профили величин $j(y)$ и $E_{\mathrm{H}}(y)$, а также магнетосопротивления $\rho_{x x}\left(\omega_{c}\right), \rho_{x y}\left(\omega_{c}\right)$.

\section{4. Чисто баллистический транспорт в умеренных магнитных полях}

Во втором и третьем баллистических подрежимах,

$$
\omega_{c} \gg \gamma^{2} W, \quad 2-\omega_{c} W \gg \gamma W,
$$

вообще говоря, динамика электронов в рамках уравнений (3) и (7) даже в главном порядке по всем малым параметрам могла бы контролироваться как действием приложенных полей и рассеянием на краях, так и рассеянием в объеме образца. Действительно, при классически малых полях, $\omega_{c} \ll \gamma$, входящих в интервал (15), скорость перераспределения импульса при межэлектронных 
столкновениях, равная $\gamma$, больше скорости изменения импульса за счет циклотронного вращения, $\omega_{c}$. Например, это отражается в том, что в знаменателе $\gamma^{2}+\omega_{c}^{2}$ формулы (8) доминирует первое слагаемое. Однако проведенный анализ выражений (8)-(14) показывает, что в обеих подобластях $\gamma^{2} W \ll \omega_{c} \ll \gamma$ и $\gamma \ll \omega_{c} \lesssim 1 / W$ диапазона (15) функция распределения электронов в главном порядке по $\gamma$ определяется рассеянием на краях и влиянием приложенных полей, а межэлектронные столкновения приводят лишь к малым поправкам.

Этот анализ основан на асимптотическом разложении аргумента экспонент в функциях $f_{ \pm}(8-10)$ во всех диапазонах $\varphi$, как для малых величин разности, $|\pi / 2-| \varphi \| \leq \omega_{c} W$, так и для больших, $|\pi / 2-| \varphi \| \gg \omega_{c} W$. Вид этих разложений зависит от отношений $\gamma / \omega_{c}$ и $\gamma^{2} W / \omega_{c}$. Когда последняя величина мала, аргумент объединенной экспоненты, полученной из произведения экспонент в формулах (8) и (9) [а также в (8) и (10)], мал во всем диапазоне углов. Сохранение лишь одного (при $\omega_{c} \gg \gamma$ ) или двух (при $\left.\gamma^{2} W \ll \omega_{c} \ll \gamma\right)$ слагаемых в разложении экспоненты в ряд приводит к тому, что функция распределения $f_{ \pm}(8)$ в главном порядке по параметру $\gamma^{2} W / \omega_{c} \ll 1\left(\right.$ и $\omega_{c} W \ll 1$ при $\left.\omega_{c} \ll \gamma\right)$ становится независимой от величины $\gamma$ при любых углах $\varphi$.

Пренебрежимую роль объемного рассеяния в главном порядке по $\gamma$ в обоих диапазонах, $\gamma^{2} W \ll \omega_{c} \ll \gamma$ и $\gamma \ll \omega_{c} \ll 1 / W$, качественно можно объяснить следующим образом. Как отмечалось в предыдущем разделе, при $\omega_{c} \gg \gamma^{2} W$ максимальный размер баллистической траектории ограничивается не длиной, связанной с объемным рассеянием, $l=1 / \gamma$, а максимальной длиной сегмента циклотронной орбиты, который можно вписать в образец, $l_{b}^{(2)}=\sqrt{R_{c} W}$ (см. рис. $\left.1, a\right)$. Следовательно, для этих обоих диапазонов $\omega_{c}$ после отражения любого электрона от края следующее его отражение от того же или противоположного края - это более вероятный процесс, нежели рассеяние в объеме на других электронах или на беспорядке. С характером динамики электронов связаны величины плотности тока вдоль оси $x$ и холловского электрического поля, получающегося из требования отсутствия тока и среднего ускорения вдоль оси $y$. Поэтому плотность тока и холловское поле в главном порядке по параметру $l_{b}^{(2)} / l \ll 1$ (и $W / l_{b}^{(2)} \ll 1$ при $\left.\omega_{c} \ll \gamma\right)$ описываются баллистическими формулами, не учитывающими рассеяние в объеме.

Таким образом, во всем диапазоне (15) выражение (8) при $\gamma=0$ дает искомую функцию распределения в главном порядке по темпу объемного рассеяния $\gamma$ при всех $у$ и $\varphi$ :

$$
\begin{aligned}
& f_{ \pm}(y, \varphi)=\tilde{c}_{l, r}+\frac{E_{0}}{\omega_{c}} \\
& \times\left\{\cos \varphi \mp \sqrt{1-\left[\sin \varphi+\omega_{c}(y \pm W / 2)\right]^{2}}\right\},
\end{aligned}
$$

где $\tilde{c}_{l, r}=E_{0} c_{l, r} / \omega_{c}^{2}$. Отметим, что при $\gamma^{2} W \ll \omega_{c} \ll \gamma$ буквальный переход к пределу $\gamma \rightarrow 0$ в функции (8) невозможен и совпадение итогового вида функции $f_{ \pm}$ при $\gamma^{2} W \ll \omega_{c} \ll \gamma$ с выражением (16) в главном порядке по параметру $\omega_{c} W \ll 1$ есть результат анализа первых двух слагаемых асимптотики общего выражения (8) по параметру $\gamma^{2} W / \omega_{c} \ll 1$ при условии $\omega_{c} W \ll 1$.

Любое решение уравнения (6) при $\gamma=0$ может быть найдено с точностью до константы, что соответствует отсутствию релаксации возмущений электронной плотности. Соответственно система алгебраических уравнений для коэффициентов $c_{l}(11)$ является вырожденной. Накладывая условие симметричности, $c_{l}+c_{r}=0$, из системы (11) мы находим:

$$
\tilde{c}_{l, r}=\mp \frac{E_{0}}{2 \omega_{c}} \frac{U-V}{2-\omega_{c} W},
$$

где $U=\arccos \left(1-\omega_{c} W\right) \quad$ и $\quad V=\left(1-\omega_{c} W\right) \sqrt{\omega_{c} W}$ $\times \sqrt{2-\omega_{c} W}$. Отметим, что вид решения (16) был получен в недавних работах [37,38], однако там не был проведен анализ области его применимости.

Как видно из выражения (16), в промежуточных магнитных полях при $\omega_{c} \sim W$ (левая часть подрежима (3)) плотность тока $j(y)$ оценивается как

$$
j(y) \sim j_{0},
$$

где $j_{0}=n_{0} E_{0} W / m-$ характерная плотность чисто баллистического тока. Уравнение (18) является следствием того, что типичные баллистические траектории, обеспечивающие главный вклад в ток $j$ при $\omega_{c} \sim 1 / W$, имеют длину $\sim W$. При тех же полях, $\omega_{c} \sim 1 / W$, функция распределения (16) приводит к следующей оценке для холловского поля:

$$
E_{\mathrm{H}}(y) \sim E_{0} .
$$

На рис. 2 мы приводим рассчитанные зависимости полного тока, $I=\int_{-W / 2}^{W / 2} d y j(y)$, усредненного холловского электрического поля, $E_{\mathrm{H}}=\int_{-W / 2}^{W / 2} d y E_{\mathrm{H}}(y) / W$, и сопротивлений $\rho_{x x}=E_{0} / I, \rho_{x y}=E_{\mathrm{H}} / I$ от параметpa $\omega_{c} W$ во всей баллистической области $0<\omega_{c}<2 / W$ при отсутствующем объемном рассеянии, $\gamma=0$. Из рис. 2 можно видеть, что оценки (18) и (19) для тока $I$ и холловского поля $E_{\mathrm{H}}$ действительно справедливы в средней части области, где $\omega_{c} \sim 1 / W$. Однако ток $I\left(\omega_{c}\right)$ расходится на обоих краях интервала, а холловское поле $E_{\mathrm{H}}\left(\omega_{c}\right)$ расходится на правом крае, $\omega_{c} W \rightarrow 2$, оставаясь конечным на левом крае, $\omega_{c} W \rightarrow 0$.

Существование конечного значения холловского поля, $E_{\mathrm{H}} \rightarrow E_{0} / \pi$, в пределе слабых полей, $\omega_{c} \rightarrow 0,-$ одно из нетривиальных проявлений баллистического характера движения электронов. Подобное поведение зависимости $\rho_{x y}\left(\omega_{c}\right)$ было получено ранее в экспериментах и численном моделировании [39] двумерных электронов в образцах с 4 контактами и было названо баллистическими аномалиями магнетосопротивления. В терминах обзора [39] зависимость $\rho_{x y}\left(\omega_{c}\right)$ на рис. $2, d$ в диапазоне $\omega_{c} W \ll 1$ представляет собой ,последнее холловское плато“. 

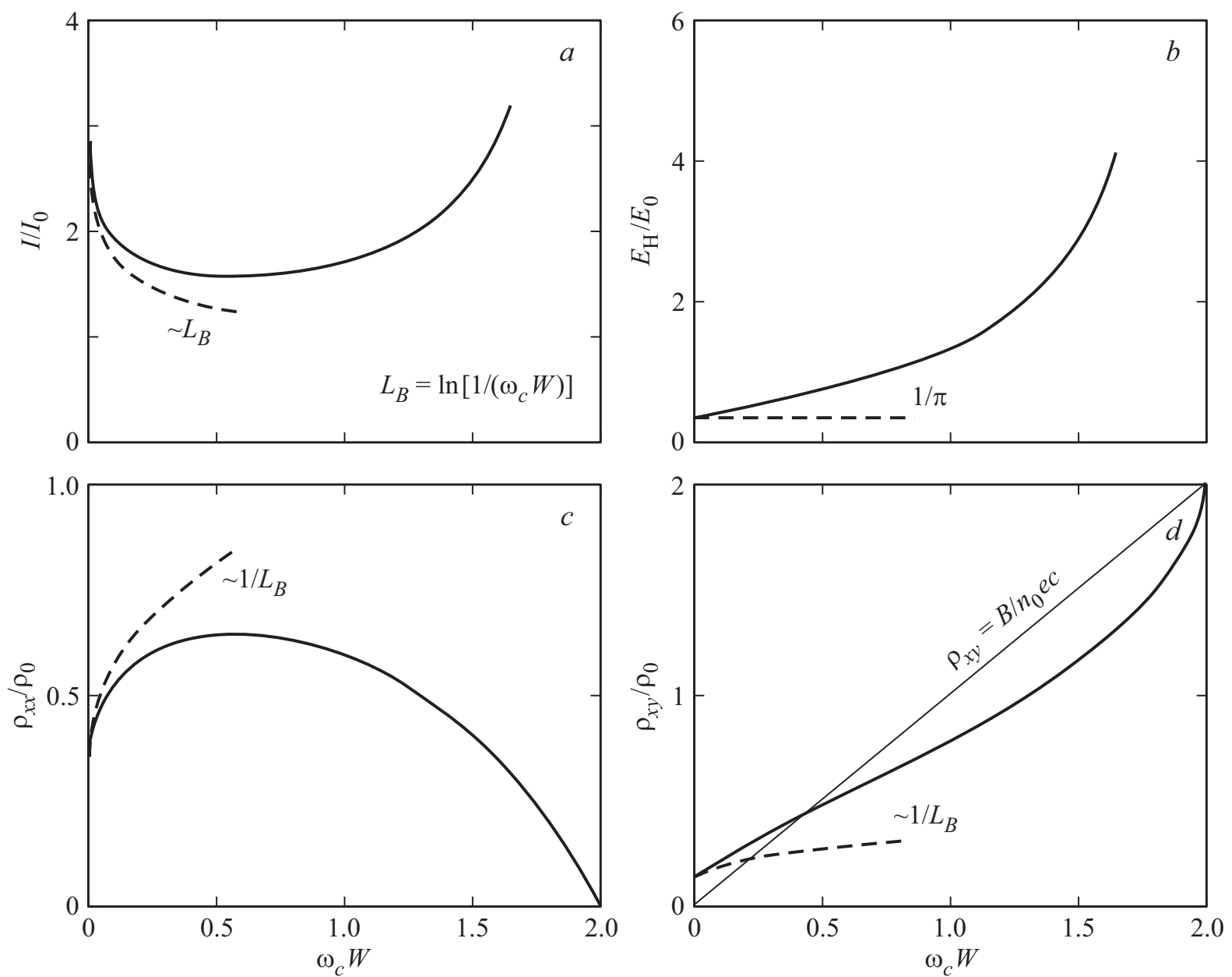

Рис. 2. Полный ток $(a)$, холловское электрическое поле $(b)$, продольное $(c)$ и холловское сопротивления $(d)$ для образца с отсутствующим объемным рассеянием, $\gamma=0$, как функции магнитного поля во втором, $0<\omega_{c} W \ll 1$, и третьем, $1 \lesssim \omega_{c} W<2$, баллистических подрежимах. Сопротивления приведены в единицах $\rho_{0}=E_{0} / j_{0}=m /\left(n_{0} W\right)$.

Далее мы получим аналитические выражения для характеристик течения в пределах $\omega_{c} W \rightarrow 0$ и $\omega_{c} W \rightarrow 2$, т. е. в подрежиме 2) и правой сингулярной части подрежима 3).

Из функции распределения (16) в пределе слабых магнитных полей, $\omega_{c} W \ll 1$, следует, что главный вклад в транспортные характеристики дают электроны, двигающиеся вдоль краев образца с углами $\varphi \approx \pm \pi / 2$. Асимптотический вид выражения (16) при углах $\sqrt{\omega_{c} W} \ll|\pi / 2-| \varphi|| \ll 1$ есть

$$
\begin{aligned}
f_{ \pm}(y, \varphi)=\tilde{c}_{l, r}+E_{0}\left[\left(y \pm \frac{W}{2}\right) \frac{\sin \varphi}{\cos \varphi}\right. \\
\\
\left.+\omega_{c} \frac{(y \pm W / 2)^{2}}{2}\left(\frac{1}{\cos \varphi}+\frac{\sin ^{2} \varphi}{\cos ^{3} \varphi}\right)\right],
\end{aligned}
$$

где

$$
\tilde{c}_{l, r}=\mp \frac{\sqrt{3} E_{0}}{3} \sqrt{\omega_{c} W^{3}}
$$

При углах $|\pi / 2-| \varphi \mid \| \lesssim \sqrt{\omega_{c} W}$ необходимо использовать полное выражение (16), в котором положено $\sin \varphi \approx \pm\left[1-(|\varphi|-\pi / 2)^{2} / 2\right]$ и $\cos \varphi \approx \pm \| \varphi|-\pi / 2|$.
Плотность тока $j(y)(4)$, отвечающая функции распределения (20), состоит из главной части, которая не зависит от координаты $y$, и малой поправки по логарифмическому параметру $L_{B}=\ln \left[1 /\left(\omega_{c} W\right)\right] \gg 1$, зависящей от $y$ :

$$
\begin{aligned}
& j(y)=j_{B}+\Delta j(y), \quad \frac{j_{B}}{j_{0}}=\frac{L_{B}}{\pi}, \\
& \frac{\Delta j(y)}{j_{0}}=f_{1}(y)+f_{2}(y)+f_{3}(y) .
\end{aligned}
$$

Функции $f_{1}(y), f_{2}(y)$ и $f_{3}(y)$ были вычислены аналитически. Они имеют схожие профили с расходящейся производной по координате у краев образца $y= \pm W / 2$ :

$$
f_{1}(y)=\frac{1}{\pi}\left(\sqrt{\frac{1}{2}+\frac{y}{W}}+\sqrt{\frac{1}{2}-\frac{y}{W}}\right)
$$

$$
\begin{aligned}
f_{2}(y)= & \frac{1}{\pi}\left\{\left(\frac{1}{2}+\frac{y}{W}\right) \ln \left[\frac{\sqrt{2}}{\sqrt{1 / 2+y / W}}\right]\right. \\
& \left.+\left(\frac{1}{2}-\frac{y}{W}\right) \ln \left[\frac{\sqrt{2}}{\sqrt{1 / 2-y / W}}\right]\right\},
\end{aligned}
$$



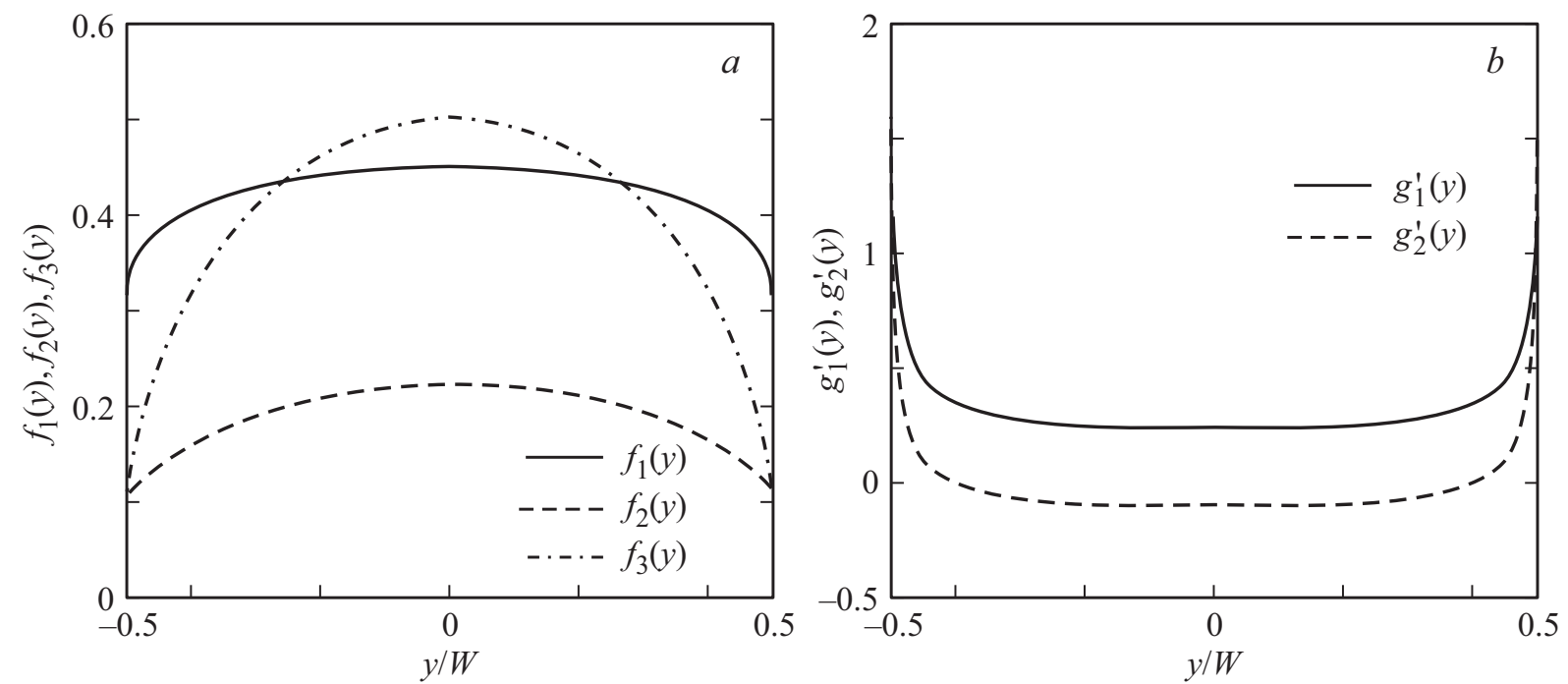

Рис. 3. Функции, определяющие профили плотности тока $(a)$ и холловского электрического поля $(b)$ согласно выражениям $(22)-(28)$ во втором баллистическом подрежиме, $(\gamma W)^{2} \ll \omega_{c} W \ll 1$, полученные в пренебрежении межчастичным рассеянием.

и

$$
\begin{aligned}
f_{3}(y)= & \frac{1}{\pi}\left\{\left(\frac{1}{2}+\frac{y}{W}\right) \ln \left[\frac{\sqrt{2}}{1-\sqrt{1 / 2-y / W}}\right]\right. \\
& \left.+\left(\frac{1}{2}-\frac{y}{W}\right) \ln \left[\frac{\sqrt{2}}{1-\sqrt{1 / 2+y / W}}\right]\right\} .
\end{aligned}
$$

Указанное сходство профилей можно увидеть из рис. $3, a$, где приведены зависимости $f_{1}(y), f_{2}(y)$ и $f_{3}(y)$.

Потенциал холловского поля (5), рассчитанный по функции распределения (20), принимает вид

$$
\phi(y)=E_{0} W\left[g_{1}(y)+g_{2}(y)\right]
$$

где

$$
g_{1}(y)=\frac{1}{2 \pi}\left(\sqrt{\frac{1}{2}+\frac{y}{W}}-\sqrt{\frac{1}{2}-\frac{y}{W}}\right)
$$

и

$$
\begin{aligned}
g_{2}(y)= & \frac{1}{2 \pi}\left\{\left(\frac{1}{2}+\frac{y}{W}\right) \ln \left[\frac{1-\sqrt{1 / 2-y / W}}{\sqrt{1 / 2+y / W}}\right]\right. \\
& \left.-\left(\frac{1}{2}-\frac{y}{W}\right) \ln \left[\frac{1-\sqrt{1 / 2+y / W}}{\sqrt{1 / 2-y / W}}\right]\right\} .
\end{aligned}
$$

Данные формулы описывают холловское поле в главном порядке по параметру $\omega_{c} W \ll 1$. Обе функции, $g_{1}(y)$ и $g_{2}(y)$, имеют расходящиеся производные вблизи краев образца, что приводит к расходимости холловского поля $E_{\mathrm{H}}(y)=-\phi^{\prime}(y)$ (см. рис. $\left.3, b\right)$.

На рис. 4 мы сравниваем результаты численного расчета профилей $j(y)$ и $E_{\mathrm{H}}(y)$, основанного на точной функции распределения (16), с аналитическими выражениями (22) и (26). Из рисунка видно, что аналитические кривые (22) и (26) корректно воспроизводят результаты численного расчета в рамках (16) для тока и холловского поля в пределе $\omega_{c} W \ll 1$. Плотность тока $j(y)$, рассчитанная точно, содержит также малую поправку $\sim j_{0}$, не зависящую от $y$, которая не приведена в формулах (22)-(25), при этом полученное численно холловское поле точно воспроизводится выражениями (22)-(26).

Из выражений $(22)-(28)$ в главном порядке по $\omega_{c} W$ получаются следующие результаты для полного тока $I$ и холловского напряжения $U_{\mathrm{H}}=\phi(W / 2)-\phi(-W / 2)$ :

$$
I=\frac{L_{B}}{\pi} j_{0} W, \quad U_{\mathrm{H}}=\frac{1}{\pi} E_{0} W .
$$

Соответственно продольное и холловское сопротивления есть

$$
\rho_{x x}=\frac{\rho_{0}}{\pi L_{B}}, \quad \rho_{x y}=\frac{\rho_{0}}{L_{B}},
$$

где $\rho_{0}=E_{0} / j_{0}=m /\left(n_{0} W\right)$. Можно видеть, что обе эти величины имеют слабую сингулярность вследствие наличия множителя $1 / L_{B}(B) \sim 1 / \ln (1 / B)$.

На рис. 5 показаны зависимости полного тока, среднего холловского поля и соответствующих сопротивлений во втором баллистическом подрежиме, $\omega_{c} W \ll 1$, от параметра $\omega_{c} W$, характеризующего величину магнитного поля. Мы сравниваем результаты численного расчета всех величин по функции распределения (16) с аналитическими результатами (29) и (30). Видно, что последние хорошо описывают численный расчет. Видно также, что сингулярности в сопротивлениях $\rho_{x x}$ и $\rho_{x y}$ при $B \rightarrow 0$ очень слабые, похожие по внешнему виду на наличие конечных пределов.

Вблизи перехода, $\gamma W \ll 2-\omega_{c} W \ll 1$ (правая часть третьего баллистического подрежима), коэффициенты $\tilde{c}_{l, r}$ (17) быстро расходятся. В главном порядке по параметру $u=2-\omega_{c} W \ll 1$ они принимают вид 

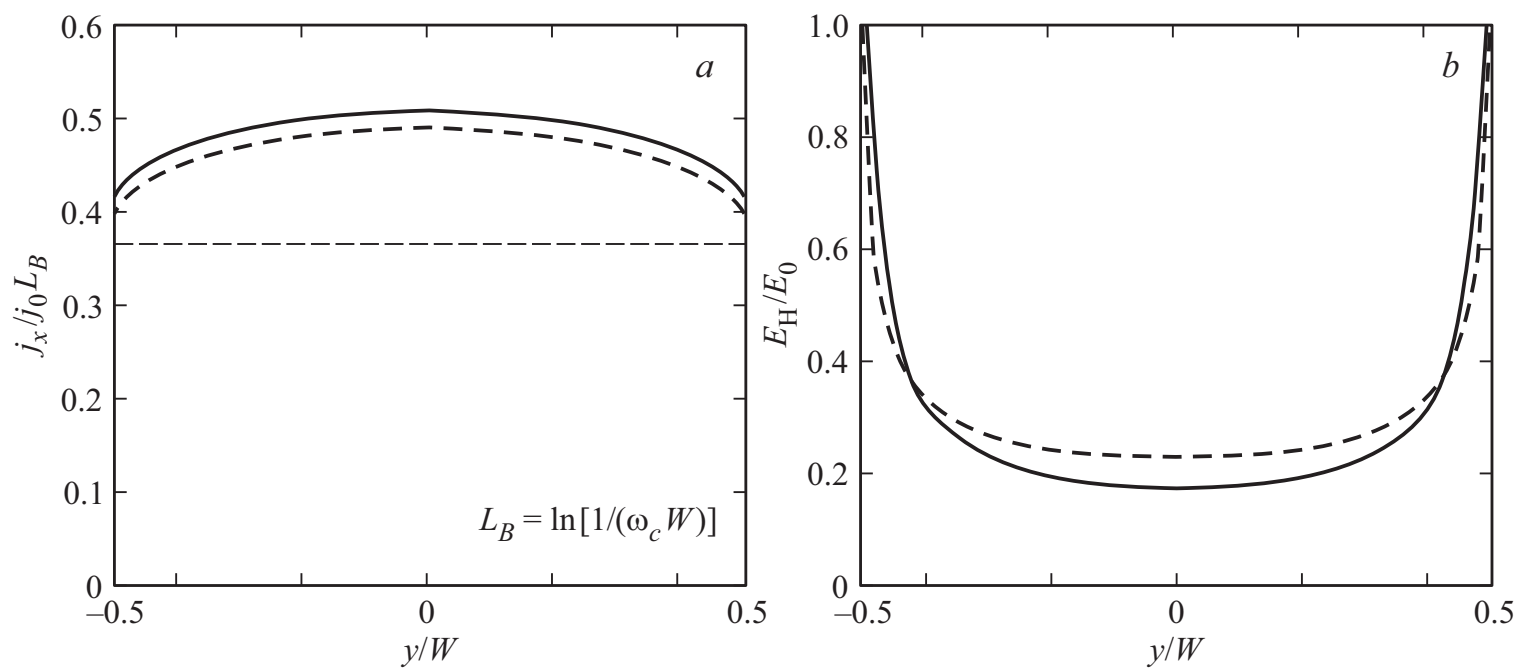

Рис. 4. Плотность тока $(a)$ и холловское поле $(b)$ при $\gamma=0$ во втором баллистическом подрежиме, $\omega_{c} W \ll 1$ (a именно $\left.\omega_{c} W=10^{-3}\right)$. Сплошные линии - результаты, полученные численно путем интегрирования функции распределения (16). Аналитические результаты (22) и (26): $a$ толстая штриховая линия; $b-$ расчет совпадает с толстой сплошной линией. Тонкая штриховая кривая на панели $a$ - главный однородный вклад в $j(y), j(y) \approx j_{B}$. Различие сплошной и толстой штриховой линий для плотности тока возникает из-за поправок по параметру $1 / L_{B}$, которые имеют порядок единицы и не учтены в формуле (22). Штриховая кривая на панели $b-$ вклад, пропорциональный производной $g_{1}^{\prime}(y)$ в (26). Он является главным вкладом в холловское поле $E_{H}(y)$, так как производная $g_{2}^{\prime}(y)$ относительно мала по сравнению с $g_{1}^{\prime}(y)$.
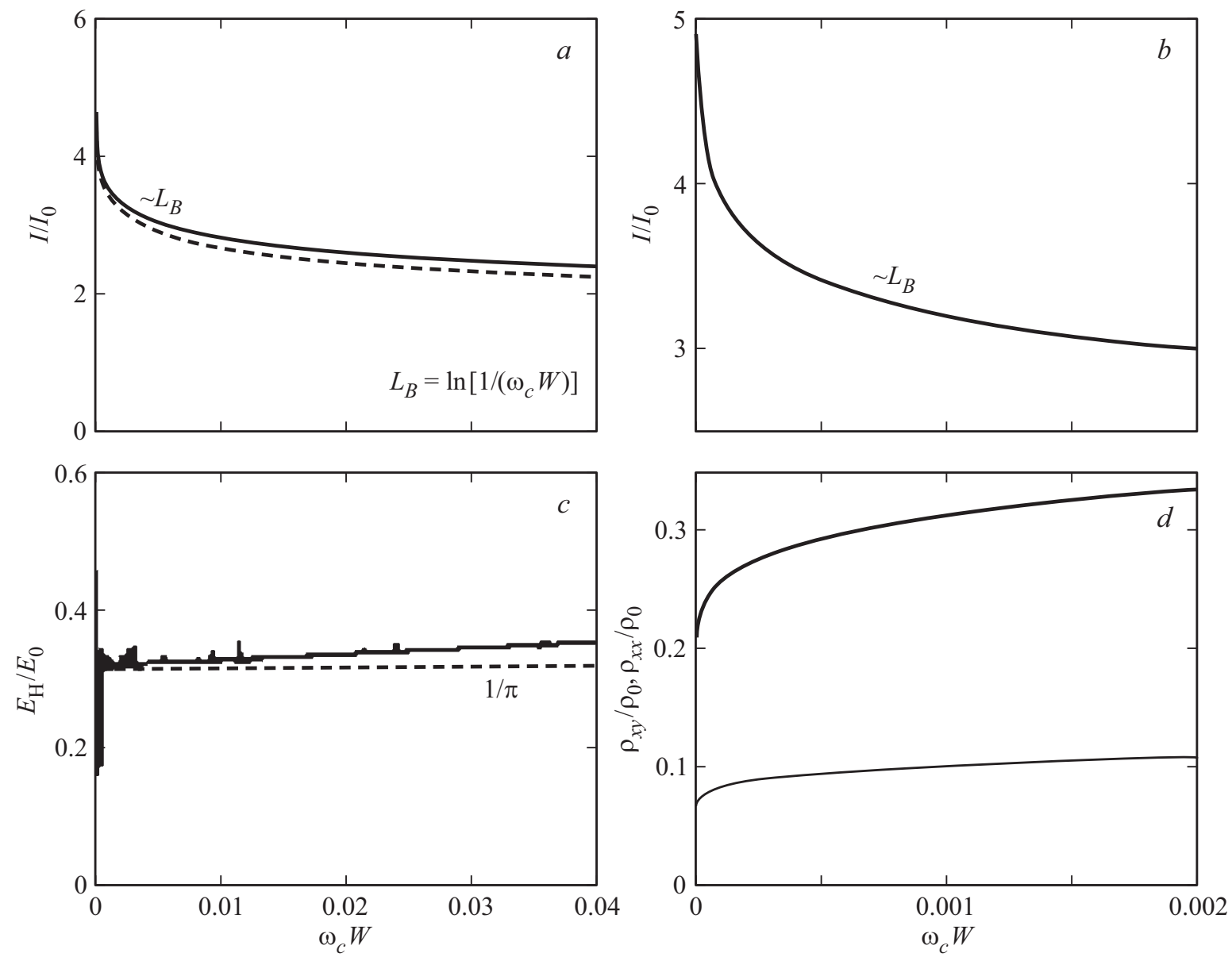

Рис. 5. Полный ток $(a, b)$, холловское электрическое поле $(c)$, продольное и холловское сопротивления $(d)$, вычисленные при $\gamma=0$, как функции магнитного поля во втором баллистическом подрежиме, $0<\omega_{c} W \ll 1$. Сплошные кривые - результаты численного расчета на основе функции распределения (16), штриховые - построены по аналитическим выражениям (29) и (30). $d$ : тонкая кривая — холловское сопротивление, толстая кривая — продольное сопротивление. 

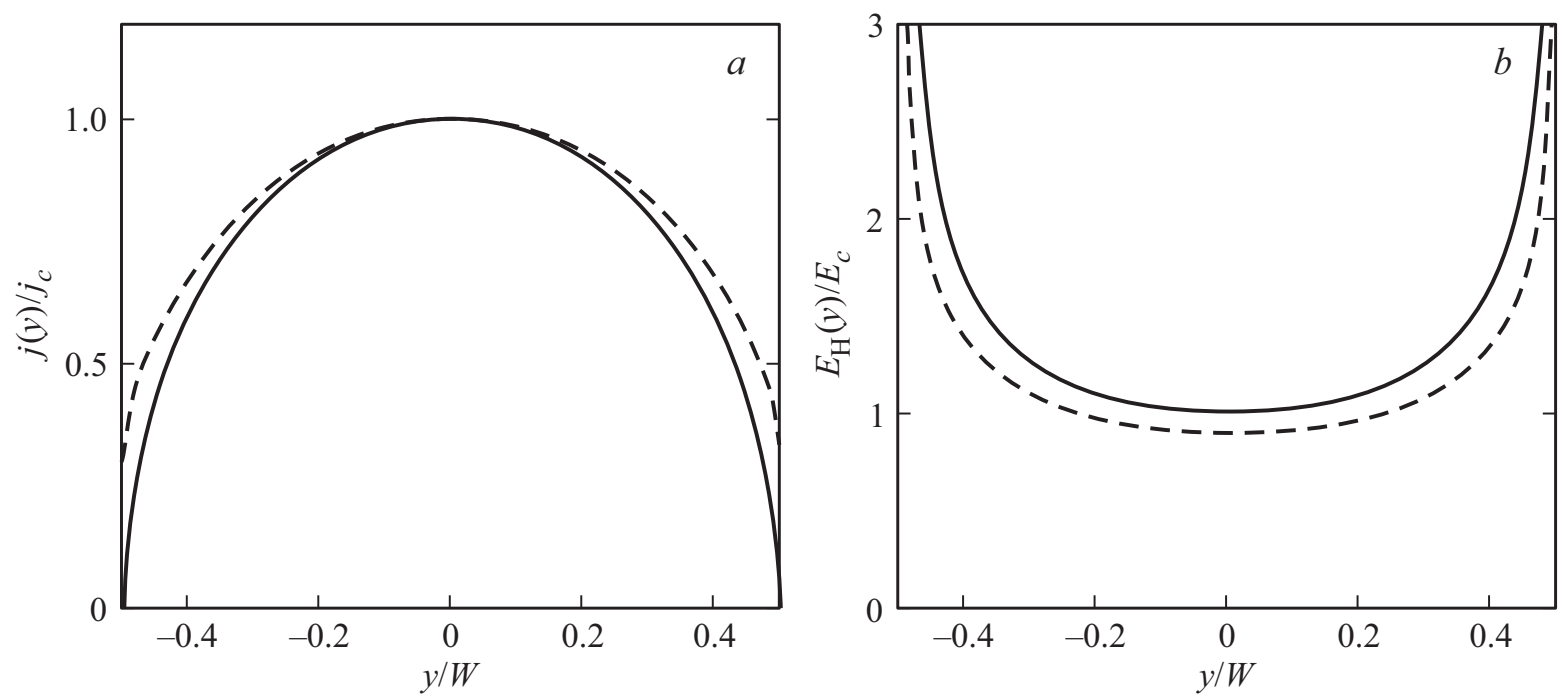

Рис. 6. Плотность тока $(a)$ и холловское электрическое поле $(b)$ в образце без объемного рассеяния, $\gamma=0$, для правой части третьего баллистического подрежима, $0<2-\omega_{c} W \ll 1$. Сплошные кривые отвечают магнитным полям, предельно близким к точке перехода, $u \rightarrow 0$, штриховые кривые соответствуют параметру $u=0.2$. Плотность тока нормирована на величину $j_{c}=2 E_{0} /\left(\omega_{c} u\right)$, холловское поле нормировано на $E_{c}=E_{0} / u$.

$\tilde{c}_{l, r}= \pm\left(\pi E_{0} /\left(2 \omega_{c} u\right)\right)$ и становятся больше других членов выражения (16). Тогда главная часть функции распределения есть [38]

$$
f_{ \pm}(y, \varphi)= \pm \frac{\pi E_{0}}{2 \omega_{c} u}
$$

Опущенные в этой формуле члены имеют порядок величины $E_{0} / \omega_{c}$.

Функция распределения (31) описывает дисбаланс между плотностями неравновесных электронов, отраженных от левого и правого краев, который нужен для компенсации компоненты тока $j_{y}$, возникающего от непосредственного действия на электроны полей $\mathbf{E}_{0}$ и В (первые два слагаемых в квадратных скобках в формуле (8)).

Расчет по формулам (4) и (5) с функцией распределения (31) позволяет получить плотность тока

$$
\begin{aligned}
j(y) & =\frac{E_{0}}{\omega_{c} u} \\
\times & {\left[\sqrt{1-\left(\omega_{c} y-\frac{u}{2}\right)^{2}}+\sqrt{1-\left(\omega_{c} y+\frac{u}{2}\right)^{2}}\right] }
\end{aligned}
$$

и электростатический потенциал холловского поля

$$
\begin{aligned}
\phi_{\mathrm{H}}(y) & =\frac{E_{0}}{2 \omega_{c} u} \\
\times & {\left[\arcsin \left(\omega_{c} y-\frac{u}{2}\right)+\arcsin \left(\omega_{c} y+\frac{u}{2}\right)\right] . }
\end{aligned}
$$

Обе величины (32) и (33), как и функция распределения (31), расходятся как $\sim 1 / u$ в магнитных полях, близких к полю перехода $\gamma W \ll 2-\omega_{c} W \ll 1$. Видно, что плотность тока имеет профиль искаженной полуокружности с очень большими производными вблизи краев образца $y= \pm W / 2$, при этом холловский потенциал имеет профиль в форме искаженного арксинуса. Эти величины для образца без рассеяния в объеме, $\gamma=0$, показаны на рис. 6 для непосредственной окрестности точки перехода, $u \rightarrow 0\left(B \rightarrow B_{c}\right)$, и для магнитного поля вблизи перехода со стороны меньших полей, $u \ll 1$ $\left(B_{c}-B \ll B_{c}\right)$.

Для полного тока и холловского напряжения в главном и следующем порядках по параметру $\sqrt{u}$ из формул (32) и (33) получаем:

$$
I=\frac{j_{0} W}{4 u}, \quad U_{\mathrm{H}}=\frac{E_{0} W}{4 u}\left(1-\frac{\sqrt{2 u}}{\pi}\right) .
$$

Соответствующие сопротивления есть

$$
\rho_{x x}=4 u \rho_{0}, \quad \rho_{x y}=\left(1-\frac{\sqrt{2 u}}{\pi}\right) \rho_{0} .
$$

Продольное сопротивление $\rho_{x x}$ как функция $u$ линейно убывает к нулю при $u \rightarrow 0$, а холловское сопротивление $\rho_{x y}$ показывает корневую расходимость при $u \rightarrow 0$. Такое поведение полного тока, холловского напряжения и сопротивлений было также нами получено путем прямых численных расчетов по функции распределения (16) (см. рис. 2).

\section{5. Обсуждение результатов}

Сопоставление результатов расчета тока и холловского поля при относительно малых $\left(\gamma^{2} W^{2} \ll \omega_{c} W \ll 1\right)$, при относительно больших, близких к критическому $\left(\gamma W \ll 2-\omega_{c} W \ll 1\right)$, а также при промежуточных 
$\left(\omega_{c} W \sim 1\right)$ магнитных полях показывает, что с ростом магнитного поля профиль плотности тока становится все более выпуклым, а у профилей холловского поля растет ширина их расходящихся особенностей у краев образца (профили $j(y)$ и $E_{\mathrm{H}}(y)$ для первого и второго случаев см. на рис. 4 и 6 , для третьего случая формы кривых $j(y)$ и $E_{\mathrm{H}}(y)$ являются промежуточными между соответствующими кривыми на рис. 4 и 6 и поэтому не приведены). Для плотности тока отношение однородной части $j(y)$ к амплитуде неоднородной части падает с ростом $\omega_{c}$ в диапазоне $\gamma^{2} W^{2} \ll \omega_{c} W \ll 1$ как логарифм, $\propto \ln \left[1 /\left(\omega_{c} W\right)\right]$ (см. формулы (22)). Последняя формула, a также численный расчет тока на основе функции распределения (16) показывают, что однородная и неоднородная части $j(y)$ сравниваются по порядку величины при $\omega_{c} W \sim 1$.

Отметим нетривиальные особенности полученных зависимостей продольного и холловского сопротивлений, $\rho_{x x}$ и $\rho_{x y}$, от магнитного поля.

Во-первых, обе эти функции являются неаналитическими в пределе $\omega_{c} W \ll 1: \rho_{x x}, \rho_{x y} \propto 1 / \ln \left[1 /\left(\omega_{c} W\right)\right]$. Сильно сингулярное поведение такого типа не встречается для омических и гидродинамических режимов транспорта и типично для баллистического транспорта, где основной вклад в ток может возникать за счет группы траекторий выделенной геометрии. В рассматриваемой системе в пределе $\omega_{c} W \ll 1$ такой группой траекторий являются сегменты циклотронных окружностей с углами скорости, близкими к направлению образца: $|\varphi| \approx \pi / 2$.

Во-вторых, примечательно, что в нижней окрестности баллистически-гидродинамического перехода при $\omega_{c} W \rightarrow 2$ холловское сопротивление $\rho_{x y}$ принимает в точности номинальное баллистическое значение $\rho_{0}$, а продольное сопротивление $\rho_{x x}$ стремится к нулю линейно по разности $B_{c}-B$ (для случая $\gamma=0$ ). Обращение в нуль величины $\rho_{x x}$ при $B \rightarrow B_{c}$ соответствует тому факту, что 2D электроны в перпендикулярном магнитном поле в полоске с шириной, равной или больше критического значения, $W \geq 2 R_{c}$, при полном отсутствии беспорядка и межэлектронного взаимодействия $(\gamma=0)$ не обладают конечным сопротивлением. В такой системе при $W \geq 2 R_{c}$ приложение внешнего поля $\mathbf{E}_{0} \| x$ в некоторый момент времени $t=0$ приводит к неограниченному росту со временем всех величин в системе: тока $j(y, t)=j_{x}(y, t)$ вдоль внешнего поля, а также тока вдоль нормали к краю образца $j_{y}(y, t)$, а следовательно, и неравновесной плотности электронов $\delta n(y, t)$ и холловского поля $E_{\mathrm{H}}(y, t)$.

Рассчитанные нами зависимости сопротивлений $\rho_{x x}(B)$ и $\rho_{x y}(B)$ в области $\gamma^{2} W^{2} \ll \omega_{c} W<2$ (рис. $2, c, d$; формулы (30) и (35)) хорошо соответствуют поведению сопротивлений, полученных в работе [36] при численном решении кинетического уравнения (1) для случая слабого межчастичного рассеяния, $\gamma W \ll 1$ (см. рис. $1, a$ и 2, $a$ работы [36]).
Совпадение особенностей полученной теоретической зависимости продольного сопротивления $\rho_{x x}(B)$ (максимум в районе $\omega_{c} W \approx 1$ и значительно меньшие величины при $\omega_{c} W \ll 1$ и $\omega_{c} W \approx 2$ ) с особенностями сопротивления, наблюдавшегося при низких температурах на высококачественных длинных образцах графена и квантовых ям GaAs (см. рис. 1, с работы [10], рис. S21, $с$ приложения к работе [11] и рис. 2 работы [19], по-видимому, свидетельствует о том, что наша теория и численная теория [36] описывают эксперименты $[10,11,18]$.

\section{6. Заключение}

Изучено баллистическое течение двумерных электронов в магнитном поле в длинных образцах (полосках) с шероховатыми краями. Показано, что в широком диапазоне магнитных полей, вплоть до критического поля перехода в гидродинамический режим, течение преимущественно определяется рассеянием электронов на краях полоски и их ускорением магнитным и электрическим полями. Рассчитаны распределения плотности тока и холловского электричского поля по сечению образца, а также продольное и холловское сопротивления как функции магнитного поля. Полученная зависимость продольного сопротивления, по-видимому, согласуется с зависимостями, наблюдавшимися в экспериментах $[10,11,18]$ на чистых образцах графена и квантовых ям GaAs в магнитных полях ниже перехода между баллистическим и гидродинамическим режимами транспорта. Представляется актуальным выполнить подробное сопоставление полученных баллистических профилей плотности тока и холловского поля с их недавними измерениями в образцах графена в экспериментах [10,11].

\section{Благодарности}

Авторы статьи благодарят А.И. Чугунова за полезные обсуждения.

\section{Финансирование работы}

Работа поддержана Российским фондом фундаментальных исследований (проект № 19-02-00999).

\section{Конфликт интересов}

Авторы заявляют, что у них нет конфликта интересов.

\section{Список литературы}

[1] R.N. Gurzhi. Sov. Phys. Uspekhi, 94, 657 (1968).

[2] M. Hruska, B. Spivak. Phys. Rev. B, 65, 033315 (2002).

[3] A.V. Andreev, S.A. Kivelson, B. Spivak. Phys. Rev. Lett., 106, 256804 (2011).

[4] P.J.W. Moll, P. Kushwaha, N. Nandi, B. Schmidt, A.P. Mackenzie. Science, 351, 1061 (2016). 
[5] D.A. Bandurin, I. Torre, R. Krishna Kumar, M. Ben Shalom, A. Tomadin, A. Principi, G.H. Auton, E. Khestanova, K.S. Novoselov, I.V. Grigorieva, L.A. Ponomarenko, A.K. Geim, M. Polini. Science, 351, 1055 (2016).

[6] L. Levitov, G. Falkovich. Nature Physics, 12, 672 (2016).

[7] A.D. Levin, G.M. Gusev, E.V. Levinson, Z.D. Kvon, A.K. Bakarov. Phys. Rev. B, 97, 245308 (2018).

[8] R. Krishna Kumar, D.A. Bandurin, F.M.D. Pellegrino, Y. Cao, A. Principi, H. Guo, G.H. Auton, M. Ben Shalom, L.A. Ponomarenko, G. Falkovich, K. Watanabe, T. Taniguchi, I.V. Grigorieva, L.S. Levitov, M. Polini, A.K. Geim. Nature Physics, 13, 1182 (2017).

[9] A.I. Berdyugin, S.G. Xu, F.M.D. Pellegrino, R. Krishna Kumar, A. Principi, I. Torre, M. Ben Shalom, T. Taniguchi, K. Watanabe, I.V. Grigorieva, M. Polini, A.K. Geim, D.A. Bandurin. Science, 364, 162 (2019).

[10] J.A. Sulpizio, L. Ella, A. Rozen, J. Birkbeck, D.J. Perello, D. Dutta, M. Ben-Shalom, T. Taniguchi, K. Watanabe, T. Holder, R. Queiroz, A. Principi, A. Stern, T. Scaffidi, A.K. Geim, S. Ilani. Nature, 576, 75 (2019).

[11] M.J.H. Ku, T.X. Zhou, Q. Li, Y.J. Shin, J.K. Shi, C. Burch, L.E. Anderson, A.T. Pierce, Y. Xie, A. Hamo, U. Vool, H. Zhang, Francesco Casola, T. Taniguchi, K. Watanabe, M.M. Fogler, P. Kim, A. Yacoby, R.L. Walsworth. Nature, 583, 537 (2020).

[12] J. Gooth, F. Menges, C. Shekhar, V. Suess, N. Kumar, Y. Sun, U. Drechsler, R. Zierold, C. Felser, B. Gotsmann. Nature Commun., 9, 4093 (2018).

[13] L. Bockhorn, P. Barthold, D. Schuh, W. Wegscheider, R.J. Haug. Phys. Rev. B, 83, 113301 (2011).

[14] A.T. Hatke, M.A. Zudov, J.L. Reno, L.N. Pfeiffer, K.W. West. Phys. Rev. B, 85, 081304 (2012).

[15] R.G. Mani, A. Kriisa, W. Wegscheider. Sci. Rep., 3, 2747 (2013).

[16] Q. Shi, P.D. Martin, Q.A. Ebner, M.A. Zudov, L.N. Pfeiffer, K.W. West. Phys. Rev. B, 89, 201301 (2014).

[17] G.M. Gusev, A.D. Levin, E.V. Levinson, A.K. Bakarov. AIP Advances, 8, 025318 (2018).

[18] G.M. Gusev, A.D. Levin, E.V. Levinson, A.K. Bakarov. Phys. Rev. B, 98, 161303 (2018).

[19] P.S. Alekseev. Phys. Rev. Lett., 117, 166601 (2016).

[20] P.S. Alekseev, A.P. Dmitriev. Phys. Rev. B, 102, 241409 (2020).

[21] Y. Dai, R.R. Du, L.N. Pfeiffer, K.W. West. Phys. Rev. Lett., 105, 246802 (2010).

[22] A.T. Hatke, M.A. Zudov, L.N. Pfeiffer, K.W. West. Phys. Rev. B, 83, 121301 (2011).

[23] M. Bialek, J. Lusakowski, M. Czapkiewicz, J. Wrobel, V. Umansky. Phys. Rev. B, 91, 045437 (2015).

[24] P.S. Alekseev. Phys. Rev. B, 98, 165440 (2018).

[25] P.S. Alekseev, A.P. Alekseeva. Phys. Rev. Lett., 123, 236801 (2019).

[26] A. Lucas. Phys. Rev. B, 95, 115425 (2017).

[27] F.M.D. Pellegrino, I. Torre, M. Polini. Phys. Rev. B, 96, 195401 (2017).

[28] P.S. Alekseev, A.P. Dmitriev, I.V. Gornyi, V.Y. Kachorovskii, M.A. Semina. Semiconductors, 51, 766 (2017).

[29] R. Moessner, P. Surowka, P. Witkowski. Phys. Rev. B, 97, 161112 (2018).

[30] P.S. Alekseev, A.P. Dmitriev, I.V. Gornyi, V.Y. Kachorovskii, B.N. Narozhny, M. Titov. Phys. Rev. B, 97, 085109 (2018).
[31] P.S. Alekseev, A.P. Dmitriev, I.V. Gornyi, V.Y. Kachorovskii, B.N. Narozhny, M. Titov. Phys. Rev. B, 98, 125111 (2018).

[32] J.Y. Khoo, I.S. Villadiego. Phys. Rev. B, 99, 075434 (2019).

[33] E.I. Kiselev, J. Schmalian. Phys. Rev. B, 99, 035430 (2019).

[34] P.S. Alekseev, M.A. Semina. Phys. Rev. B, 98, 165412 (2018).

[35] P.S. Alekseev, M.A. Semina. Phys. Rev. B, 100, 125419 (2019).

[36] T. Scaffidi, N. Nandi, B. Schmidt, A.P. Mackenzie, J.E. Moore. Phys. Rev. Lett., 118, 226601 (2017).

[37] T. Holder, R. Queiroz, T. Scaffidi, N. Silberstein, A. Rozen, J.A. Sulpizio, L. Ella, S. Ilani, A. Stern. Phys. Rev. B, 100, 245305 (2019).

[38] A.N. Afanasiev, P.S. Aleksseev, A.A. Greshnov, M.A. Semina. arXiv:2010.01642 (2021)

[39] C.W.J. Beenakker, H. van Houten. Solid State Phys., 44, 1 (1991).

Редактор Л.В. Шаронова

\section{Ballistic flow of two-dimensional electrons in a magnetic field}

\author{
A.N. Afanasiev, P.S. Alekseev, A.A. Greshnov, \\ M.A. Semina \\ loffe Institute, \\ 194021 St. Petersburg, Russia
}

Abstract In conductors with a very small density of defects, electrons at low temperatures collide predominantly with a sample edges. Therefore, the ballistic regime of charge and heat transport is realized. The application of a perpendicular magnetic field substantially modifies the character of ballistic transport. For the case of two-dimensional (2D) electrons in the magnetic fields corresponding to the diameter of the cyclotron trajectories smaller than the sample width a hydrodynamic transport regime is formed. In the latter regime, the flow is mainly controlled by rare electronelectron collisions, which determine the viscosity effect. In this work, we study the ballistic flow of $2 \mathrm{D}$ electrons in long samples in magnetic fields up to the critical field of the transition to the hydrodynamic regime. From solution of the kinetic equation, we obtain analytical formulas for the profiles of the current density and the Hall electric field far and near the ballistic-hydrodynamic transition as well as for the longitudinal and Hall resistances in these ranges. Our theoretical results, apparently, describe the observed longitudinal resistance of pure graphene samples in the diapason of magnetic fields below the ballistic-hydrodynamic transition. 